\title{
Edge detection of fuzzy droplet image based on combination of Canny operator and morphology operation
}

\author{
Xu Yingqiang, Xu Zhiniu, Li Yang \\ Power Engineering Department, North China Electric Power University, Baoding 071003, China \\ Yingqiang9@163.com
}

Keywords: image processing, edge detection, remove a small target, morphological operations.

\begin{abstract}
On the basis of Canny operator on image processing, The edge extraction of the fuzzy droplet images is carried out by combination of removing a small target and morphological open operation .First, Use edge detection operator to detect the image edge preliminary, and then call remove a small target which belongs to MATLAB internal function to filtering the results of the edge detection, and finally with morphological operations for final processing. The results showed that: the combination of Canny operator, open operation and remove a small target works much better than the classical Canny operator.
\end{abstract}

\section{Introduction}

Image edge extraction is widely used in science and engineering. But the commercial contact angle measuring instrument is adopting the conventional edge detection algorithm, it is difficult to extract the edge of fuzzy image accurately and difficult to be practical. In this paper, a more accurate and practical edge detection algorithm is studied, its significance lies in avoiding using the naked eye to determine the droplet edge point, saving the workload greatly and it is important for the successful completion of hydrophobic Detection

\section{Mathematical morphology}

Mathematical morphology is a lattice theory and topology built on the basis of image analysis disciplines, it is the basic theory of mathematical morphology image processing. The basic operations include: binary erosion and dilation, the binary opening and closing operation, skeleton extraction, limit corrosion, hit-miss transform, morphological gradient, Top-hat transformation, particle analysis, watershed transformation and so on.

(1) The basic mathematical morphology operation. a.Expansion: if the background point of the object to be detected is contact with the boundary of the object, its borders will merge these background points to external expansion. Expansion is mathematically defined as the collection operation, if the object $A$ to be measured is expanded by the structural elements $B$, then recorded as dilation $(\mathrm{A}, \mathrm{B})=A \oplus B$. The principle is: the structural element $\mathrm{B}$ translates a distance obtains $\mathrm{Ba}$, if $\mathrm{Ba}$ hit the object $\mathrm{A}$ which is under test, we have a note of this point. All points satisfy the above conditions consist of a set which is called the result of the expansion by structural element $B$. Consider two binary images $\mathrm{A}$ and $\mathrm{B}$. Their prospects are black and background is white. Another $\mathrm{fA}$ and $\mathrm{fB}$ represents a collection of individual foreground pixels. dilation $(\mathrm{A}, \mathrm{B})=\{\mathrm{a}+\mathrm{b} \mid \mathrm{a} \in \mathrm{A}, \mathrm{b} \in$ B \}.Such as:

$$
\begin{aligned}
& \mathrm{fA}=\{(2,8),(3,6),(4),(5,6),(6,4),(7,6),(8,8)\} \\
& \mathrm{fB}=\{(0,0),(0,1)\} \\
& \text { dilation }(\mathrm{A}, \mathrm{B})=\{(2,8),(2,9),(3,6),(3,7),(4),(4,5),(5,6),(5,7),(6,4),(6,5),(7,6),(7,7),
\end{aligned}
$$

b.Corrosion: It is an elimination of the boundary points, the process is making boundaries inward. Corrosion can be seen as dual operation of expansion. The principle is: the structural element $\mathrm{B}$ translates a distance and obtains $\mathrm{Ba}$, if $\mathrm{Ba}$ contains in the object $\mathrm{A}$ to be measured, we have a note of this point, All points satisfy the above conditions consist of a set which is called the result of the 
corrosion by structural element $\mathrm{B}$. Also consider two images $\mathrm{A}$ and $\mathrm{B}$, definition of corrosion operation is: corrosion $(\mathrm{A}, \mathrm{B})=\{\mathrm{a} \mid(\mathrm{a}+\mathrm{b}) \in \mathrm{A}, \mathrm{a} \in \mathrm{A}, \mathrm{b} \in \mathrm{B}\}$.

The nature of the expansion, corrosion operation:

Exchange law: dilation $(\mathrm{A}, \mathrm{B})=\operatorname{dilation}(\mathrm{B}, \mathrm{A})$

Combining law: dilation (dilation $(A, B), C)=$ dilation $(A$, dilation $(B, C)$ )

Union: dilation $(\mathrm{A}, \mathrm{B} \cup \mathrm{C})=$ dilation $(\mathrm{A}, \mathrm{B}) \cup$ dilation $(\mathrm{A}, \mathrm{C})$

Growth: if $A$ belongs to $B$ then dilation $(A, K)$ belongs to dilation $(B, K)$

(2) Combined operation based on corrosion and expansion

1. Open operation: if structure element $B$ applys open operation for the object $A$, this operation to $\mathrm{A}$ by $\mathrm{B}$ is corroding first and then use $\mathrm{B}$ to expand the results: $A \circ B=(A \otimes B) \oplus B$

2. Close operation: if structure element $B$ applys close operation for the object $A$, this operation to $\mathrm{A}$ by $\mathrm{B}$ is expanding first and then use $\mathrm{B}$ to corrode the results: $A \bullet B=(A \oplus B) \otimes B$

3. Edge detection of morphological image based on MATLAB

MATLAB image processing toolbox has the functions of image transformation, mathematical morphology, image enhancement and image restoration. We can call the image processing operator directly in MATLAB and use its function to remove the small targets. The processing of the fuzzy droplets image is as below.

The concrete steps of the algorithm are as follows :

Step One: Using Canny operator to extract the edge of the droplet image initially.

Step Two: Call MATLAB internal function bwareaopen (remove small targets) to do further processing after Step One .

Step Three: use morphological Open operation for image filtering after Step Two, we will get the final results.

\section{The experimental results}

Place a drop of water on the surface of the silicone rubber specimen, use digital camera to get the original image. The following results are obtained after the three steps mentioned above.

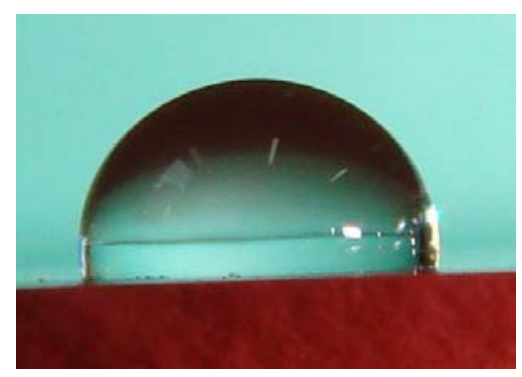

$(1)$

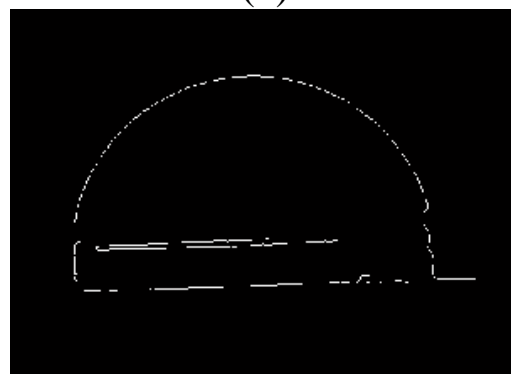

(3)

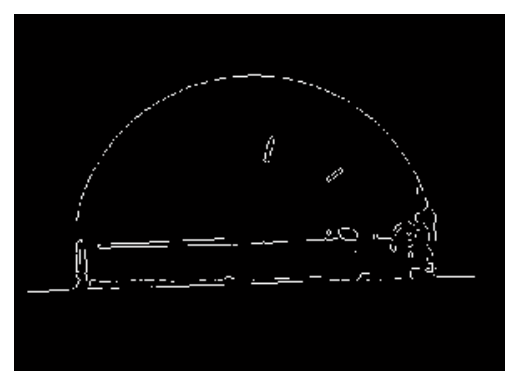

(2)

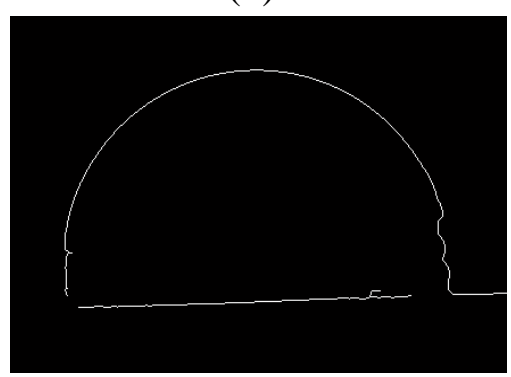

(4)

(1) Original image.

(2) Image after Canny operator.

(3) Image after the combination of Canny operator and remove small targets.

(4) Image after the combination of Canny operator , remove small targets and Open operation. 


\section{Result analysis}

(1) Since the Canny operator uses a large scale for filtering, the detection results are missing some edges.

(2) Call MATLAB internal function bwareaopen to do further processing after Step One. The results indicate that: the combination of Canny operator and remove small targets to process image gets less edge noises than by using the Canny operator alone

(3) Compared with the previous two steps, the third step uses the combination of Canny operator, remove small targets and Open operation to deal with the image of the droplets, the edge we last get is more complete, clearer, more precise edge positioning.

\section{Summary}

In the image analysis and processing, the role of edge detection is crucial. The effect of dealing with fuzzy drop image by mathematical morphology is often determined by the threshold and shape of the selected structure elements. When the shape of the selected structure element is certain, the pixel threshold range is weak and the noise is weak, but it can detect good edge details. The pixel threshold range is strong and the noise is strong, but there may be some loss of the edges. Therefore, How to select the appropriate threshold for image processing will be the future research direction in practical application.

\section{References}

[1] JIN Pengfei. An improved Sobel edge detection algorithm [J] .Journal of Applied Optics, 2008, 29 (4): 625-628.

[2] WANG Na, LI Xia. a new improved Canny edge detection algorithm [J] .Shenzhen University: Science \& Engineering, 2005,22 (2): 149-153.

[3] YANG Fan. digital image processing and analysis [M]. Beijing: Beijing University of Aeronautics and Astronautics Press, 2007: 220-221.

[4] SUN Yinghui .Edge Detection Canny operator [J] .Anshan Normal University, 2010,12 (2): 48-50.

[5] LIN Hui, ZHAO Changsheng, SHU Ning.edge detection and evaluation of Canny Operator [J]. Heilongjiang Institute of Technology: Natural Science Edition, 2003,17 (2): 3-6.

[6] YU Songhuang, ZHOU Yuanhua, Zhang Rui. image processing [M]. Shanghai: Shanghai Jiaotong University Press, 2007. 